\title{
Der Atomausstieg ist kein vorrangig technisches Problem
}

Die Würfel zur künftigen deutschen Energiepolitik sind gefallen: Im Lichte der Atomkatastrophe von Fukushima wird Deutschland bis zum Jahr 2022 aus der Atomkraft aussteigen. Doch wie ist dieser Ausstieg zu schaffen? Meine, im Folgenden zu erläuternde These ist, dass der Umbau unseres Energiesystems keine vorrangig technische Herausforderung ist und dass das deutsche Beispiel eine wichtige Signalwirkung für die Energie- und Klimadebatte weltweit haben wird.

Dass wir heute - anders als 1986 nach der Katastrophe von Tschernobyl - den Ausstieg aus der Atomenergie wagen, hat mit den gewaltigen Fortschritten bei den regenerativen Energien in diesen 25 Jahren zu tun. 1986 stand die damals weltweit größte 3-Megawatt (MW)-Windtestanlage GROWIAN kurz vor dem Aus, die Wirkungsgrade von Photovoltaikanlagen waren vernachlässigbar. Heute dagegen werden 4-MW-Anlagen für den Offshore-Windbetrieb in Serie gefertigt, weit über 25 Gigawatt (GW) installierte Windkraftleistung erbringen einen signifikanten Anteil an der deutschen Stromversorgung. 35 \% regenerativer Energieanteil an unserer Stromversorgung bis 2020 sind ein realistisches politisches Ziel; bis zu $100 \%$ im Jahr 2050 erscheinen technisch ohne Probleme machbar. Möglich wird dies dadurch, dass auch in den angegliederten technischen Bereichen wichtige Fortschritte erzielt wurden: z.B. bei der aktiven Netzsteuerung (Smart Grids) oder bei Speichertechnologien.

Die wichtigen Fragen, die uns in den kommenden Jahren beschäftigen werden, liegen auf anderen Ebenen:

- Sie sind ökonomischer Natur: Welche Auswirkungen hat der Umbau auf die Strompreise? Was bedeutet dies für die Wettbewerbsfähigkeit bestimmter Industriebranchen in Deutschland? Was sind mögliche soziale Folgen weiter steigender Strompreise?

- Sie sind gesellschaftlich-institutioneller Natur: Wie schaffen wir die nötige Legitimität und Beteiligung bei dem jetzt anstehenden Infrastrukturausbau - von neuen Windparks bis zu neuen Stromnetzen und Energiespeichern?

- Sie sind zivilisatorischer Natur: Gelingen uns die nötigen Energie-Effizienz- und Einsparziele, die allen Umbauszenarien zugrunde liegen? Bisher verfehlen wir diese Effizienzsteigerungen gesamtwirtschaftlich - nicht weil uns effizientere Geräte fehlen, sondern weil unser Lebensstil durch Mehrverbrauch die erzielten Effizienzvorteile wieder zunichte macht. Ein Blick in den Elektronikgerätebestand (vom Fernseher über Computer bis zum Handy) eines Haushaltes im Jahr 1986 im Vergleich zum Jahr 2011 macht das sehr schnell deutlich.

Auf diesen Ebenen liegen die eigentlichen Herausforderungen - und hier zeigt sich auch die besondere Bedeutung des deutschen Weges. Denn über das Für und Wider der isolierten Gefahren der Atomtechnologie kann man lange diskutieren. Entscheidend am deutschen Weg ist, dass eine der weltweit führenden Industrienationen Flagge zeigt, aus dieser Technologie auszusteigen und die „Stromlücke“ durch regenerative Energien mehr als auszugleichen. Wenn dies Deutschland ökonomisch und gesellschaftlich erfolgreich gelingt, dann ist das ein Signal für alle Industrie- und Schwellenländer, dann hat das deutsche Beispiel Ausstrahlungskraft auf die globale Energiedebatte: Der Ausstieg aus ökologisch bedenklichen Großkraftwerksstrukturen (insbesondere Atomund Kohle) ist möglich, ohne die industrielle Basis und die ökonomische Entwicklung zu gefährden.

Übrigens liegt genau darin auch die gewaltige ökonomische Chance des deutschen Ausstiegs. Die in Deutschland entstehenden (System-)Lösungen werden zu einem noch wichtigeren Exportprodukt der deutschen Volkwirtschaft in den kommenden Jahren werden.

Um die Herausforderung zu meistern, bedarf es einer guten ökonomischen, gesellschaftlichen und wissenschaftlichen Einbettung des Umbaus:

Ökonomisch kommt den richtigen Preissignalen eine zentrale Bedeutung zu. „Preise müssen die ökologische Wahrheit sagen.“ Nur dann werden die wirtschaftlichen Akteure ihr Handeln entsprechend ausrichten. Die geeignete Fortführung der 1999 eingeführten ökologischen Steuerreform wäre hierzu ein möglicher Weg. Durch moderate, aber kontinuierliche Preisanstiege würde Erwartungssicherheit für Konsumenten und Investoren geschaffen und zudem der Faktor Arbeit kostenmäßig entlastet. Durch geeignete Ausnahmeregeln ließe sich die Wettbewerbsfähigkeit energieintensiver, aber effizienter Branchen erhalten.

Gesellschaftlich brauchen wir Experimente mit neuen Planungs- und Beteiligungsverfahren. Die Erfahrungen mit dem Schlichtungsprozess für Stuttgart 21 oder mit der für den Atomausstieg eingesetzten Ethikkommission stimmen optimistisch. Ähnlich wie wir neue Technologien breit erproben, brauchen wir mehr Experimentierkultur auch im institutionellen Bereich.

Das hat auch wissenschaftlich Konsequenzen. Die Forschungsprogramme der Zukunft werden weniger technologisch und mehr gesellschafts- und kulturwissenschaftlich geprägt sein müssen. Es bedarf einer neuen „Transformationswissenschaft“, wie es der Wissenschaftliche Beirat Globale Umweltveränderungen (WBGU) der Bundesregierung in seinem aktuellen Hauptgutachten ausgedrückt hat.

So werden wir den Umbau des Energiesystems schaffen.

Uwe Schneidewind, Prof. Dr., ist Präsident des Wuppertal Institut für Klima, Umwelt, Energie. Arbeitsschwerpunkte: Innovationsmanagement und Nachhaltigkeit.

e-mail: Uwe.Schneidewind@wupperinst.org 